\title{
Variation in working memory capacity and episodic memory: Examining the importance of encoding specificity
}

\author{
Nash Unsworth • Gene A. Brewer • Gregory J. Spillers
}

Published online: 13 September 2011

(C) Psychonomic Society, Inc. 2011

\begin{abstract}
In the present study, we examined the extent to which encoding specificity influences the relation between individual differences in working memory capacity (WMC) and episodic recall. Participants performed a paired associates cued recall task in which a rhyme or a semantic judgment was made during encoding. During recall participants were presented with the cue word along with either a rhyme or semantic cue. Across both rhyme and semantic conditions, encoding and retrieval conditions either matched or mismatched. When encoding and retrieval conditions matched, high WMC individuals outperformed low WMC individuals. When encoding and retrieval conditions mismatched, high and low WMC individuals performed equivalently. Importantly, this occurred because high WMC individuals were hurt more than low WMC individuals when conditions mismatched. These results demonstrate the importance of encoding specificity in the relation between WMC and episodic recall as well as of unifying prior work that has demonstrated that high WMC individuals are hurt more in some recall conditions than are low WMC individuals.
\end{abstract}

Keywords Working memory· Individual differences in memory capacity

N. Unsworth $(\bowtie)$

Department of Psychology, University of Oregon,

Eugene, OR 97403, USA

e-mail: nashu@uoregon.edu

G. A. Brewer

Arizona State University,

Phoenix, AZ, USA

G. J. Spillers

University of Oregon,

Eugene, OR, USA
Working memory, and individual differences in working memory capacity (WMC), has long been shown to be an important predictor of higher order cognitive abilities, such as intelligence. Recent work suggests that retrieval of information from long-term memory is also an important component of working memory and part of the reason for individual differences in WMC (Unsworth \& Engle, 2007). In particular, recent correlational work has suggested that measures of working memory are moderately to strongly related with measures of long-term memory (Mogle, Lovett, Stawski, \& Sliwinski, 2008; Unsworth, Brewer, \& Spillers, 2009), suggesting an intimate link between working and long-term memory. Importantly, this research suggests that the shared variance between working memory and long-term memory partially accounts for the relation between working memory and intelligence (Unsworth et al., 2009).

Despite initial evidence for a relation between WMC and long-term memory abilities, the reason for the relation is still not fully understood. It is possible that the relation is due to differences in encoding, differences in retrieval, or a combination in both such that there are differences in encoding-retrieval interactions. Our goal in the present study was to better examine individual differences in WMC and their relation to long-term memory by manipulating aspects of encoding and retrieval and examining encodingretrieval interactions in the hopes of shedding light on the relation between WMC and long-term memory abilities.

Prior work has suggested that WMC differences in episodic memory performance reflect a joint combination of strategic encoding and contextual-retrieval (Unsworth \& Spillers, 2010). In terms of strategic encoding differences, we suggested that high WMC individuals are better at engaging in strategic encoding processes (e.g., rehearsal, imagery, grouping, etc.) during the presentation of items 
than are low WMC individuals (Bailey, Dunlosky, \& Kane, 2008; McNamara \& Scott, 2001; Turley-Ames \& Whitfield, 2003; Unsworth \& Spillers, 2010). Important evidence for this claim comes from studies that suggest that high WMC individuals are more likely to report using encoding strategies, and these strategies partially account for the relation between WMC and long-term memory abilities (Bailey et al., 2008; Unsworth \& Spillers, 2010). We also suggested that high WMC individuals are better at using contextual cues/probes to focus the search on correct items during retrieval (Unsworth \& Spillers, 2010). Evidence for this claim comes from recent studies that have found that under incidental encoding conditions, in which strategic encoding processes are minimized, high WMC individuals recall more items than do low WMC individuals (Unsworth \& Spillers, 2010). Thus, the relation between WMC and long-term memory abilities seems to be due to a joint combination of encoding and retrieval processes.

To account for these results, we (Unsworth \& Spillers, 2010) suggested that the relation between WMC and longterm memory abilities could be due to differences in the ability to reinstate the encoding context at retrieval similar to encoding specificity (Tulving \& Thomson, 1973). Specifically, encoding specificity refers to the notion that recall of an event depends on the interaction between features of the encoded event and features of the retrieval cue. Thus, if attention is paid to rhyme features of a word at encoding, a rhyme cue will be more effective at retrieval than a semantic cue (Fisher \& Craik, 1977). In terms of WMC, we have argued that high WMC individuals utilize a more effective retrieval plan whereby they are better than low WMC individuals at encoding information as distinct, which then results in more specific cues/probes at retrieval. In terms of encoding specificity, this suggests that when the encoding context and the retrieval context match, high WMC individuals should outperform low WMC individuals as is typically seen.

Importantly, the flip side should also be true in that when the encoding context and the retrieval context do not match, high WMC individuals should be hurt worse than low WMC individuals. Put simply, if high WMC individuals actively attempt to take in information in a specific way so that the information can be more easily retrieved later (as part of their retrieval plan), then any disruption of matching encoding and retrieval processes should hurt their performance (i.e., strategy disruption, Basden \& Basden, 1995; Cokely, Kelley, \& Gilchrist, 2006). By contrast, if low WMC individuals are less likely to both actively attempt to encode information and purposely try to recapitulate encoding processes at retrieval (i.e., their retrieval plan is less focused), then they should be hurt less by any disruption of matching encoding and retrieval processes.
The idea that WMC interacts with encoding specificity to the extent that high WMC individuals are actually hurt more than low WMC individuals when there is a mismatch between encoding and retrieval conditions is consistent with prior results in the literature in which high WMC individuals have been shown to be hurt more by part-list cuing interference (Cokely et al., 2006) and by directed forgetting instructions (Delaney \& Sahakyan, 2007). In both of these studies, it was found that the performance of high WMC individuals was hurt more than the performance of low WMC individuals when there was a mismatch between encoding and retrieval contexts. This will be discussed more thoroughly in the Discussion section, but for the present purposes, note that perhaps the link between these studies is due to the fact that high WMC individuals are more sensitive to manipulations of encoding specificity than low WMC individuals because of differences in strategic encoding and retrieval processes. This notion that WMC interacts with encoding specificity makes the counter-intuitive prediction that high WMC individuals will be hurt more than low WMC individuals in a longterm memory task. Critically, this prediction goes against the naive assumption that high WMC individuals will always outperform low WMC individuals.

To directly examine the prediction that encoding specificity is important for the relation between WMC and episodic recall, we had high and low WMC individuals perform a paired associates cued-recall task in which encoding and retrieval conditions were manipulated. Specifically, a variant of a task employed by Fisher and Craik (1977; their Experiment 2) was used in order to examine both levels of processing (Craik \& Lockhart, 1972) and encoding specificity (Tulving \& Thomson, 1973) within the same paradigm. Levels-of-processing is the notion that information is remembered as a function of how deeply it is processed with semantic processing typically leading to deeper processing than rhyme processing and hence better memory when semantic features are attended to. In the paired associates task, participants were given two words that either rhymed or were semantically related. During encoding, participants made either rhyme judgments or semantic judgments. At retrieval, participants were presented with a rhyme cue or a semantic cue that either matched or mismatched the encoding condition. Thus, levels-of-processing was manipulated by requiring participants to make either a shallow or a deep judgment for each pair, and encoding specificity was manipulated by having the encoding and retrieval conditions either match (rhyme encoding and rhyme retrieval) or mismatch (rhyme encoding and semantic retrieval). If variation in WMC is simply due to how well information is encoded, then we should see that forcing low WMC individuals to encode information deeply should alleviate their memory deficits as compared 
with high WMC individuals. If, however, WMC differences are due to differences in encoding specificity, then both encoding and retrieval conditions should matter. That is, if high WMC individuals demonstrate more sensitivity to encoding specificity than low WMC individuals, then we should see that high WMC individuals outperform low WMC individuals when encoding and retrieval conditions match, but that high WMC individuals are hurt more (they demonstrate a greater drop in performance) when encoding and retrieval conditions mismatch.

\section{Method}

Participants were recruited from the participant pool at the University of Georgia. Individuals were selected on the basis of a $z$-score composite of three complex span tasks. Only participants falling in the upper (high WMC individuals) and lower (low WMC individuals) quartiles of the composite distribution were selected.

Operation span Participants solved a series of math operations while trying to remember a set of unrelated letters. Participants were required to solve a math operation, and after solving the operation, they were presented with a letter for $1 \mathrm{~s}$. Immediately after the letter was presented, the next operation was presented. At recall, letters from the current set were recalled in the correct order by clicking on the appropriate letters. For all of the span measures, items were scored if the item was correct and in the correct position. The score was the number of correct items in the correct position.

Reading span Participants were required to read sentences while trying to remember a set of unrelated letters. Participants read a sentence and determined whether the sentence made sense or not. Half of the sentences made sense, whereas the other half did not. Nonsense sentences were made by simply changing one word from an otherwise normal sentence. After participants gave their responses, they were presented with a letter for $1 \mathrm{~s}$. At recall, letters from the current set were recalled in the correct order by clicking on the appropriate letters. The same scoring procedure as Ospan was used.

Symmetry span Participants were required to recall sequences of red squares within a matrix while performing a symmetry-judgment task. In the symmetry-judgment task, participants were shown an $8 \times 8$ matrix with some squares filled in black. Participants decided whether the design was symmetrical about its vertical axis. The pattern was symmetrical half of the time. Immediately after determining whether the pattern was symmetrical, partici- pants were presented with a $4 \times 4$ matrix with one of the cells filled in red for $650 \mathrm{~ms}$. At recall, participants recalled the sequence of red-square locations in the preceding displays, in the order they appeared by clicking on the cells of an empty matrix. The same scoring procedure as Ospan was used.

For the composite score, scores for each of the three complex span tasks were $z$-transformed for each participant. These $z$-scores were then averaged together, and quartiles were computed from the averaged distribution. Participants were 26 high $\mathrm{WMC}$ individuals $(z-\mathrm{WMC}=.88, \mathrm{SD}=.16)$ and 25 low WMC individuals $(z-\mathrm{WMC}=-1.17, \mathrm{SD}=.32)$, as determined by the composite measure.

\section{Cued recall procedure}

Participants were presented with a list of 48 word pairs. Each word pair consisted of one word in capital letters, the target word, and above it in lowercase letters was the cue word. The cue word either rhymed with or was semantically related to the target word. During the presentation of each word pair, participants were required to make either a rhyme judgment or a semantic judgment to ensure that attention was focused on whether the words rhymed or were semantically related. For each judgment, participants indicated on a four point scale (e.g., $1=$ don't rhyme; $4=$ rhyme) how well they thought the words either rhymed or were semantically related by pressing the appropriate keys (1-4) on the keyboard. The questions always led to a positive response so that for the rhyme questions, the cue and target always rhymed, and for the semantic questions, the cue and target were always semantically associated. Each pair was presented for $5 \mathrm{~s}$ each, during which time participants were required to make their judgments. Participants were instructed to learn the target words but to use the context words as aids to remember the target words (Fisher \& Craik, 1977). After the presentation of the last word, participants were given a cued recall test. Participants were presented - along with the cue word - a statement indicating that the target word either rhymed with the cue word or was semantically related to the cue word. The cue word was related to only one target word. For example, participants might see "Rhymes with dog" for the rhyme cue condition or they might see "Associated with dog" in the semantic cue condition. Participants were instructed to recall the word that was presented with the cue word. Participants were instructed to type in the target word and then to press ENTER to indicate their response. Participants had $5 \mathrm{~s}$ to type in the corresponding word. Similar to Fisher and Craik, the test order always matched the presentation order so that the study-test lag was constant for all items. 
For each participant, half of the target words were presented in the rhyme context, and the other half in the semantic encoding context. Likewise, half of the retrieval cues were rhyme, and half were semantically related. This resulted in four conditions: rhyme encoding and rhyme retrieval (condition RR), rhyme encoding and semantic retrieval (condition RS), semantic encoding and rhyme retrieval (condition SR), and semantic encoding and semantic retrieval (condition SS). Each condition consisted of 12 trials per participant. Each response was hand scored to ensure that responses that were accurate, but simply misspelled words were credited as being correct. Doing this eliminated the possibility that any differences between high and low WMC individuals could be due to mere differences in spelling ability.

\section{Results}

Proportion correct on the cued recall task was examined with a 2 (encoding condition: rhyme vs. semantic) x 2 (retrieval condition: rhyme vs. semantic) x 2 (WMC: high vs. low) mixed ANOVA with WMC as the between-subjects factor. The ANOVA yielded a main effect of encoding condition, $F(1,49)=267.28, M S E=.01, p<.01, \eta_{\mathrm{p}}{ }^{2}=.85$, in which more items were recalled in the semantic condition $(M=.47, S E=.01)$ than in the rhyme condition $(M=.22, S E=.01)$. This finding represents the standard levels of processing effect seen numerous times. The ANOVA also yielded a main effect of retrieval condition, $F(1$, $49)=60.65, M S E=.01, p<.01, \eta_{\mathrm{p}}{ }^{2}=.55$, so that more items were recalled in the semantic condition $(M=.41, S E=$ $.01)$ than in the rhyme condition $(M=.29, S E=.01)$. Importantly, these two factors interacted $F(1,49)=$ 267.05, MSE $=.01, p<.01, \eta_{\mathrm{p}}{ }^{2}=.85$. Shown in Table 1 is proportion correct as a function of encoding and retrieval conditions. As can be seen, performance was best when encoding and retrieval conditions matched than when they mismatched, and this effect was maximal in the semantic encoding and semantic retrieval condition. This result replicates Fisher and Craik (1977), demonstrating encoding specificity and levels of processing effects simultaneously in this task.

Table 1 Proportion correct as a function of encoding and retrieval conditions

\begin{tabular}{llc}
\hline & \multicolumn{2}{l}{ Retrieval Condition } \\
\hline Encoding Condition & Rhyme & Semantic \\
Rhyme & $.28(.02)$ & $.17(.01)$ \\
Semantic & $.29(.01)$ & $.65(.02)$ \\
\hline
\end{tabular}

Note. Values in parentheses represent one standard error of the mean
In terms of individuals differences, there was a main effect of WMC, $F(1,49)=12.21, M S E=.02, p<.01, \eta_{\mathrm{p}}{ }^{2}=.20$, in which more items were recalled by high WMC individuals $(M=.38, S E=.01)$ than by low WMC individuals $(M=.32$, $S E=.01)$. Variation in WMC did not interact with either encoding or retrieval conditions, both $F_{\mathrm{s}}<1$. Thus, levels of processing did not interact with WMC. ${ }^{1}$ Importantly, however, there was a three-way interaction between encoding condition, retrieval condition, and WMC, $F(1,49)=$ $10.44, M S E=.01, p<.01, \eta_{\mathrm{p}}{ }^{2}=.18$. As is shown in Fig. 1a, high and low WMC individuals differ in performance only when the encoding and retrieval conditions match. When there is a mismatch between encoding and retrieval conditions, high and low WMC individuals demonstrate equivalent performance. This can be more readily seen in Fig. 1b, in which the two matching conditions have been combined, and the two mismatching conditions have been combined. As can be seen, there was no WMC difference in the mismatch condition, $t(49)=.96, p>.33, \eta^{2}=.02$. But in the match condition, there is a strong WMC difference, $t(49)=3.86, p<.01, \eta^{2}=.23$. Thus, high WMC individuals seem to outperform low WMC individuals only when encoding and retrieval conditions match. Furthermore, and in line with the main prediction of the study, when encoding and retrieval conditions mismatch, high WMC individuals demonstrate a greater drop in performance than low WMC individuals. That is, as is shown in Fig. 1b, the drop from the match condition to the mismatch condition is greater for high WMC individuals $(M=.28, S E=.02)$ than for low WMC individual $(M=.19$, $S E=.02)$. Thus, high WMC individuals are hurt more by a mismatch between encoding and retrieval conditions than are low WMC individuals.

\section{Discussion}

In the present study, we examined the extent to which the relation between episodic long-term memory and WMC is dependent on encoding specificity. In particular, we examined the counter-intuitive prediction that high WMC individuals would be hurt more than low WMC individuals when encoding and retrieval contexts do not match. Consistent with this prediction, the results showed that when encoding and retrieval contexts match, high WMC individuals outperformed low WMC individuals, as is typically seen. However, when there was a mismatch between encoding and retrieval contexts, high and low

\footnotetext{
${ }^{1}$ There were no differences between high and low WMC individuals in their rhyme or semantic ratings during the encoding phase (both $p \mathrm{~s}>.85$ ). Furthermore, an examination of the overall distribution of responses suggested that high and low WMC individuals made very similar ratings during the encoding phase.
} 

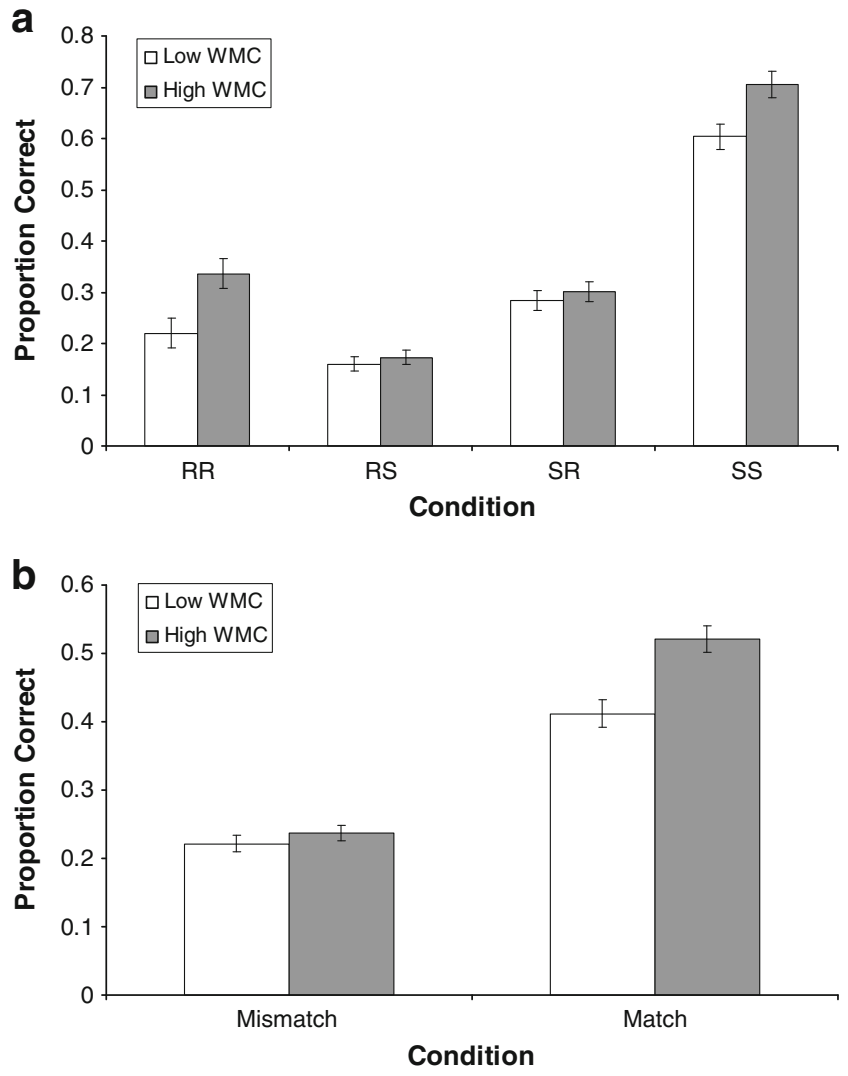

Fig. 1 a Proportion correct as a function of encoding and retrieval conditions and working memory capacity (WMC). b Proportion correct as a function of matching or mismatching encoding and retrieval conditions and working memory capacity (WMC) Error bars reflect one standard error of the mean

WMC individuals performed equivalently. Thus, simply mismatching encoding and retrieval contexts served to equate performance for high and low WMC individuals. Importantly, this result was due to the fact that high WMC individuals demonstrated a greater drop in performance than did low WMC individuals. In normal recall conditions in which encoding and retrieval contexts match, high WMC individuals are better able to strategically encode information and utilize a retrieval plan that capitalizes on that encoded information than are low WMC individuals. When encoding and retrieval contexts do not match, the ability to use encoded information disrupts performance more for high WMC individuals than for low WMC individuals. Thus, those processes that typically aid high WMC individuals' superior recall can also lead to greater decrements in performance when those processes cannot be adequately utilized.

As was noted previously, the notion that high WMC individuals are hurt more when encoding and retrieval contexts do not match helps to tie together several studies reporting some situations wherein high WMC individuals demonstrate worse memory performance than low WMC individuals. For instance, Cokely et al. (2006) examined variation in WMC and part-list cuing. They found that high WMC individuals were hurt more by part-list cues than were low WMC individuals. However, when high and low WMC individuals were required to engage in an elaborative encoding strategy, high and low WMC individuals were hurt to the same extent by part-list cues. In accounting for these results, Cokely et al. suggested that high WMC individuals utilized elaborative encoding strategies to encode the information and, at retrieval, when presented with cues that did not match how the information was encoded, the performance of high WMC individuals suffered (i.e., strategy disruption, Basden \& Basden, 1995). Because low WMC individuals were less likely to use elaborative encoding strategies and were less likely to utilize those strategies as part of their retrieval plans, they were disrupted less by the presence of the part-list cues. However, when low WMC individuals were forced to utilize elaborative encoding strategies, their performance suffered to the same extent as that of high WMC individuals. Like in the present study, this result suggests that high WMC individuals will be disrupted more than low WMC individuals when they cannot recapitulate strategic encoding processes at retrieval.

In a similar vein, Delaney and Sahakyan (2007; see also Aslan, Zellner, \& Bauml, 2010), examined variation in WMC and directed forgetting. In this study, participants were presented with a list of words (List 1). Following the presentation of this list, they were instructed to keep remembering the words (remember condition), to forget the words (forget condition), or to engage in a context change manipulation in which they were instructed to imagine walking around their parents' houses (context change condition). All participants were then presented with a second list of words to remember. Consistent with prior work, Delaney and Sahakyan found that fewer List 1 items were recalled in both the forget and context change conditions, as compared with the remember condition. Importantly, this effect interacted with WMC such that in the remember condition, high WMC individuals recalled more items than low WMC individuals. However, in both the forget and context change conditions, high WMC individuals actually recalled fewer items than did low WMC individuals. Similar to the results of the present study and those of Cokely et al., (2006) high WMC individuals outperformed low WMC individuals when the encoding and retrieval contexts matched (remember condition), but the performance of high WMC individuals suffered when the encoding and retrieval contexts did not match (forget and context change conditions).

Finally, in a recent study, we (Unsworth, Spillers, \& Brewer, 2011) examined variation in WMC and categorical free and cued recall. Important for the present study was 
that in one condition, participants were given a list of categorical words presented in random order and were asked to free recall the words. In another condition, a different list of categorical words was presented in random order, and at recall, participants were given category labels as cues and were instructed to recall only the words from the specified category. Reanalyzing the data suggested that there was a significant WMC $\mathrm{x}$ retrieval interaction, $p<.01$, $\eta_{\mathrm{p}}{ }^{2}=.11$, such that the performance of high WMC individuals dropped in the cued recall condition as compared with that in the free recall condition $(p<.01)$, but there was no difference for low WMC individuals $(p>.37)$. This result suggests that, given that high WMC individuals expected a free recall test, they may have engaged in strategies at encoding to link the words together, and these strategies were actually detrimental to their performance when presented with cues at recall. Low WMC individuals were less likely to use elaborative strategies at encoding; thus, the shift to cued recall likely had less of an effect on their overall performance, similar to what was reported by Cokely et al. (2006).

Thus, across four very different studies (part-list cuing, directed forgetting, categorical free and cued recall, and encoding retrieval interactions in paired associates recall) similar results arise in terms of individual differences in WMC. In each case, high WMC individuals are hurt more than low WMC individuals by various manipulations that lead to a mismatch between encoding and retrieval contexts. When encoding and retrieval contexts match, high WMC individuals outperform low WMC individuals. Given that in most prior studies of WMC and episodic recall encoding and retrieval contexts have matched, it would seem that high WMC individuals typically outperform low WMC individuals. However, in those few situations in which encoding and retrieval contexts do not match, it would seem that high WMC individuals are hurt more than low WMC individuals.

These results point to the importance of encoding specificity in understanding individual differences in WMC and episodic recall. The present results help tie together prior work by suggesting that differences in encoding specificity may explain why the performance of high WMC individuals suffers more than that of low WMC individuals from various manipulations. Furthermore, the notion that encoding specificity plays an important role in the relation between WMC and episodic recall predicts that when encoding and retrieval contexts do not match, high WMC individuals should be affected more than low WMC individuals. Importantly, this should primarily arise when participants actively encode information and utilize a retrieval plan that aids in the strategic search for encoded items. That is, it is not simply the case that high WMC individuals will be hurt with any mismatch between encoding and retrieval contexts, but that high WMC individuals will be hurt when the specific strategies they utilized at encoding are disrupted and are no longer effective at retrieval. Future work should be directed at examining how encoding specificity influences the relation between WMC and episodic memory.

Author Note Correspondence concerning this article should be sent to N. Unsworth, Department of Psychology, University of Oregon, Eugene, OR 97403 (e-mail nashu@uoregon.edu).

\section{References}

Aslan, A., Zellner, M., \& Bauml, K. H. (2010). Working memory capacity predicts listwise directed forgetting in adults and children. Memory, 18, 442-450.

Bailey, H., Dunlosky, J., \& Kane, M. J. (2008). Why does working memory span predict complex cognition? Testing the strategy affordance hypothesis. Memory \& Cognition, 36, 1383-1390.

Basden, D. R., \& Basden, B. H. (1995). Some tests of the strategy disruption interpretation of part-list cuing inhibition. Journal of Experimental Psychology. Learning, Memory, and Cognition, 21, $1656-1669$.

Cokely, E. T., Kelley, C. M., \& Gilchrist, A. H. (2006). Sources of individual differences in working memory: Contributions of strategy to capacity. Psychonomic Bulletin \& Review, 13, 991-997.

Craik, F. I. M., \& Lockhart, R. S. (1972). Levels of processing: A framework for memory research. Journal of Verbal Learning and Verbal Behavior, 11, 671-684.

Delaney, P. F., \& Sahakyan, L. (2007). Unexpected costs of high working memory capacity following directed forgetting and contextual change manipulations. Memory and Cognition, 35, 1074-1082.

Fisher, R. P., \& Craik, F. I. M. (1977). Interaction between encoding and retrieval operations in cued recall. Journal of Experimental Psychology: Human Learning and Memory, 3, 701-711.

McNamara, D. S., \& Scott, J. L. (2001). Working memory capacity and strategy use. Memory \& Cognition, 29, 10-17.

Mogle, J. A., Lovett, B. J., Stawski, R. S., \& Sliwinski, M. J. (2008). What's so special about working memory? An examination of the relationship among working memory, secondary memory, and fluid intelligence. Psychological Science, 19, 1071-1077.

Tulving, E., \& Thomson, D. (1973). Encoding specificity and retrieval processes in episodic memory. Psychological Review, 80, 352-372.

Turley-Ames, K. J., \& Whitfield, M. M. (2003). Strategy training and working memory task performance. Journal of Memory and Language, 49, 446-468.

Unsworth, N., Brewer, G. A., \& Spillers, G. J. (2009). There's more to the working memory-fluid intelligence relationship than just secondary memory. Psychonomic Bulletin \& Review, 16, 931-937.

Unsworth, N., \& Engle, R. W. (2007). The nature of individual differences in working memory capacity: Active maintenance in primary memory and controlled search from secondary memory. Psychological Review, 114, 104-132.

Unsworth, N., \& Spillers, G. J. (2010). Variation in working memory capacity and episodic recall: The contributions of strategic encoding and contextual retrieval. Psychonomic Bulletin \& Review, 17, 200-205.

Unsworth, N., Spillers, G.J., \& Brewer, G.A. (2011). Working memory capacity and retrieval limitations from long-term memory: An examination of differences in accessibility. Manuscript submitted for publication 